International Journal of Wavelets, Multiresolution

and Information Processing

Vol. 15, No. 6 (2017) 1750053 [21 pages)

(C) The Author(s)

DOI: $10.1142 / \mathrm{S} 0219691317500539$

\title{
A bio-inspired two-scale complementarity evaluation method for image fusion
}

\author{
Yanxiang $\mathrm{Hu}^{*}$ and Bo Zhang \\ College of Computer and Information Engineering \\ Tianjin Normal University, No. 393, Binshui West Road \\ Xiqing District, Tianjin 300387, P. R. China \\ *huyanxiang@mail.tjnu.edu.cn \\ Received 4 March 2017 \\ Revised 26 June 2017 \\ Accepted 3 July 2017 \\ Published 22 August 2017
}

\begin{abstract}
A bio-inspired two-scale image complementarity evaluation method is proposed. This novel multi-scale method provides a promising alternative for the performance assessment of image fusion algorithms. Moreover, it can also be used to compare and analyze the multi-scale difference of raw images. Two metrics are presented and used to assess the complementarity of fusion images in non-subsampled contourlet transform (NSCT) domains: visual saliency differences (VSDs) at the coarse scales and detail similarities (DSs) at the fine scales. Visual attention mechanism (VAM)-based saliency maps are combined with NSCT low-pass subbands to compute the VSDs, and linear correlation and contrast consistency-based DSs are compared in NSCT band-pass subbands. Five main multi-scale transform (MST)-based fusion algorithms were compared by using 30 groups of raw images that consist of four types of fusion images. Effects of NSCT filters and decomposition levels on evaluation results are discussed in detail. Furthermore, a group of color multi-exposure fusion images were also taken as examples to evaluate the complementarity of raw images. Experimental results demonstrate the effectiveness of the proposed method, especially for MST-based image fusion algorithms.
\end{abstract}

Keywords: Image fusion; visual complementarity; visual attention mechanism; visual saliency; non-subsampled contourlet transform.

AMS Subject Classification: 34E13, 62H35

\section{Introduction}

As image sensing techniques continue to develop, more and more image data from various kinds of image sensors have become available. Due to the limitations of electronic imaging technologies, such as spectrum range, dynamic range, temporal and spatial resolution, etc., images captured by a single image sensor can only partially

This is an Open Access article published by World Scientific Publishing Company. It is distributed under the terms of the Creative Commons Attribution 4.0 (CC-BY) License. Further distribution of this work is permitted, provided the original work is properly cited. 
reveal the content of the observed scenes. Therefore, image fusion technology, which integrates different raw images to obtain more inferences than can be derived from a single image, has now been used successfully in many imaging applications. $\frac{132712720}{120}$

Among hundreds of variations of image fusion techniques, multi-scale transform (MST)-based pixel-level fusion algorithms have become the most important category in recent years $\frac{19}{19}$ A variety of MST tools, including various pyramid decompositions and smooth filters, ${ }^{20123}$ various discrete wavelet transforms (DWTs) $)^{2612331319}$ and stationary wavelet transforms (SWTs), 15 ridgelet transform, 211 curvelet transform (CVT), subsampled contourlet transform (NSCT) ${ }^{29116114}$ have been employed in image fusion. Other new methods of image representation and analysis, such as dictionary learning and sparse representation, ${ }^{29124}$ dense scale invariant feature transform (SIFT),$\frac{32}{22}$ etc., have also been investigated in depth.

In an MST-based fusion algorithm, each raw image is first decomposed into a low-frequency subband and a group of high-frequency subbands with different scales and orientations. Then the fusion rules select the subband coefficients that have higher saliency to construct the corresponding fusion subband coefficients. These resulting coefficients are then used to reconstruct fusion results through inverse transforms. Some papers $17 \llbracket 19$ have analyzed the performance of various MST-based pixel level fusion methods in depth. Reference 17 investigated the fusion performance of DWT, SWT, Dual-Tree Complex Wavelet Transform (DTCWT), CVT, CT and NSCT with different decomposition levels and filters, for multi-focus fusion, medical image fusion and infrared-visible fusion. The fusion quality was evaluated with several commonly used metrics that are also employed in Ref. 15. Their works showed that three shift-invariant MSTs, SWT, DTCWT and NSCT, have clear advantages over DWT and CT. It was also found that short filters usually perform better, and the appropriate number of decomposition levels is usually four.

Despite the fact that many studies have been carried out on the assessment of image fusion algorithms, the existing evaluation metrics cannot fully reveal the characteristics of MST-based fusion algorithms. A basic drawback of these wellaccepted fusion metrics, such as that introduced in detail in Ref. 25, is the attempt to use a holistic metric to sum up the fusion performance within different scales. Hence, a targeted evaluation on MST-based algorithms cannot be carried out by these metrics from the perspective of multi-resolution analysis.

In this paper, the complementarity of fusion images is defined as the visual otherness between raw images or sources and their combinations. Necessities of this research lay in: 1. Assessing the fusion quality at different spatial scales. The capabilities to extract information from different scales can be taken as a quality index for MST-based fusion algorithms. A fusion algorithm would show different capabilities when used for fused images with different types. 2. Evaluating the multi-scale visual differences of raw images, and quantitatively selecting the best characteristic distributions of raw images. 3. Guiding the development of algorithm fusion methods. It has been demonstrated that combinations of fusion algorithms 
can improve the fusion quality effectively ${ }^{7}$ So our metrics can provide quantitative comparison results for MST algorithm combinations.

Inspired by the multi-stage and multi-scale perception characteristics of human visual system (HVS), the proposed method evaluates the complementarity of fusion images from two scales: VSDs in low-frequency scales and detail DSs in highfrequency scales. In our method, NSCT which has been demonstrated to be the best method for multi-resolution image decomposition, 17 is employed as the MST tool. At the same time VAM is used to extract regional saliencies. To the best of the authors' knowledge, our method is the first one that evaluates fusion quality from the perspective of VAM.

The rest of the paper is structured as follows. Section 2 briefly introduces the basic principles of the VAM. Section 3 explains the proposed method in detail. Section 4 discusses experimental results and Sec. 5 concludes the paper.

\section{VAM and Visual Saliency}

VAM, which means that different regions in an image have different visual stimuli, is one of the most important features of HVS. Humans would first focus their attention on those regions with the highest degree of saliency in the pre-attention stage, and then allocate rational computing resources for further recognitions and interpretations. VAM has been intensively researched over the past two decades, and many VAM models have been presented, $\frac{45}{4}$ including Itti's bottom-up data-driven model, $\frac{12}{12}$ spectral residual model, $\frac{10}{10}$ etc.

Saliency measurements and coefficient combinations are two key factors in fusion methods. The absolute amplitudes of MST coefficients, local energy or local standard derivations are usually used to determine the regional salient degrees. Because the visual saliency is influenced by many factors, these simple saliency metrics are not reasonable. In VAM, visual stimuli are accessed by taking into account multiple visual characteristics, and a complex multi-scale round-center subtraction strategy is employed to generate comprehensive results. The resulting visual saliency maps (VSMs) contain the stimuli intensity of every pixel, and a larger intensity value indicates a higher visual saliency. This stimulus intensity combined with NSCT low-frequency coefficients is used as the saliency index to evaluate the complementarity between the sources and their fusion results at coarse scales. A fusion algorithm based on this idea has been presented in Ref. 11. In this paper, the classical Itti's model and an implementation ${ }^{[8}$ were employed. Other VAM models, such as the spectral residual model in Ref. 10, also have been tested and they present similar performances with Itti's model in our experiments.

The computation process of Itti's model contains two stages. Feature maps of intensity, color and orientation are extracted from an image at first, using complex round-center subtraction strategy with multi-channel and multi-scale filter banks. This gradient strategy assigns larger saliency to the locations where more rapid changes take place. Then these partial feature maps are combined to form a global 


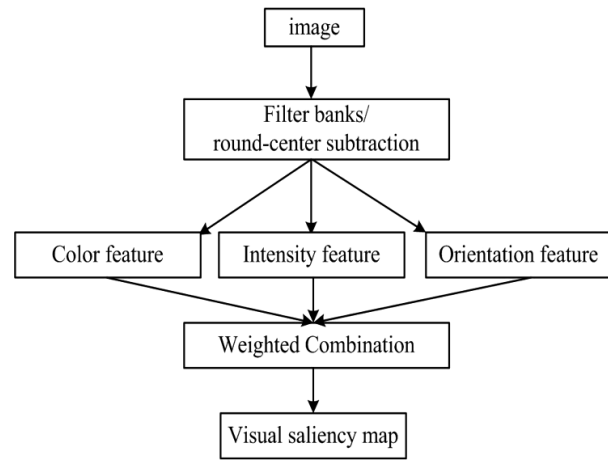

(a)
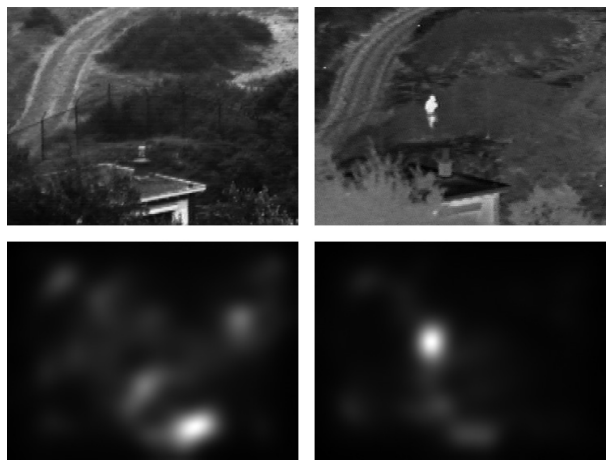

(b)

Fig. 1. The computational flow of VAM and a pair of examples. (a) The computational flow of VAM, (b) Infrared-visible images and corresponding VSMs.

saliency map. This model has been widely used in many image applications. Furthermore, it has become the basis of later VAM models and a standard benchmark for performance comparisons. Figure 1 shows the computational flow of VAM and a pair of examples.

\section{Two-Scale Complementarity Evaluation Method}

In this paper, NSCT is employed as the MST tool. NSCT has been demonstrated to have more flexible multi-resolution, anisotropy and directional expansion for image fusion. 17 Because the number of direction decompositions at each level can be differing, NSCT is much more valuable for contour and detail representation. For color image fusion, the complementarity is assessed in the HSV space. Three components are processed separately and then combined to form a whole index. The schematic diagram of the proposed algorithm is shown in Fig. 2 .

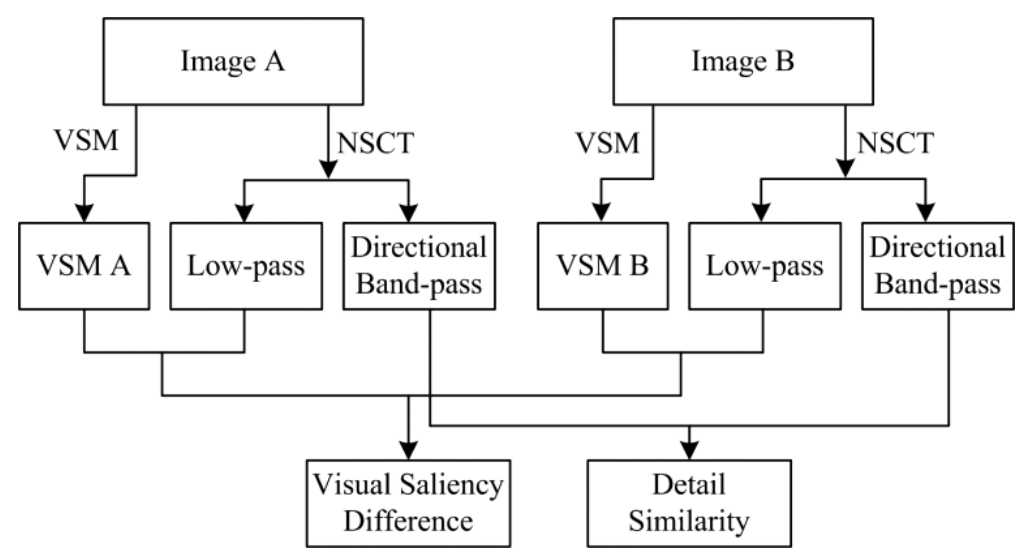

Fig. 2. The schematic diagram of the proposed algorithm. 
After NSCT, raw images $A$ and $B$ are decomposed into low-pass subbands $L_{A}, L_{B}$, and band-pass subbands $H_{A}^{l, l k}$ and $H_{B}^{l, l k}$, where $l$ denotes the $l$ th decomposition level and $l k$ denotes the $k$ th directional band-pass subband at the $l$ th decomposition level.

\subsection{Visual saliency difference based on VSM and NSCT low-pass subbands}

Visual saliency difference VSD is computed as follows:

(1) Compute VSMs $\operatorname{VSM}_{A}$ and $\mathrm{VSM}_{B}$ of images $A$ and $B$, respectively. $\mathrm{VSM}_{A}$ and $\mathrm{VSM}_{B}$ have the same size with $A$ and $B$.

(2) Compute the dot product of VSM and the corresponding NSCT low-pass subband $L$, denoted as VSM_L

$$
\mathrm{VSM}_{-} L_{A / B}=\mathrm{VSM}_{A / B} \cdot \times L_{A / B} \text {. }
$$

(3) Compute the saliency matching degree $M_{A, B}$ at each pixel and VSD between $A$ and $B$

$$
\begin{aligned}
M_{A, B}(i, j) & =\left|V_{\text {SSM_L }}(i, j)-\operatorname{VSM}_{A} L_{B}(i, j)\right|, \\
\operatorname{VSD}_{A, B}= & \frac{\sum_{j=1}^{N} \sum_{i=1}^{N} M_{A, B}(i, j)}{(M \times N)},
\end{aligned}
$$

where $M$ and $N$ are the sizes of the raw images. Here the dot product of VSMs and low-pass subbands of NSCT is used to calculate $M_{A, B}(i, j)$ and $\operatorname{VSD}_{A, B}$. This can effectively avoid the edge blurring caused by VSM, which may hide rapid intensity changes.

\subsection{DS based on directional band-pass subbands of NSCT}

To remove the interference caused by luminance changes, detail similarity (DS) is extracted and contrasted in all directional band-pass subbands of NSCT. Inspired by Ref. 28 , DS at location $(i, j)$ between $A$ and $B$ is defined as

$$
\operatorname{DS}_{A, B}(i, j)=\frac{2\left|\sigma_{x} \sigma_{y}\right|}{\sigma_{x}^{2}+\sigma_{y}^{2}} \times \frac{\left|\sigma_{x y}\right|}{\left|\sigma_{x} \sigma_{y}\right|}=\left.\frac{2\left|\sigma_{x y}\right|}{\sigma_{x}^{2}+\sigma_{y}^{2}}\right|_{w},
$$

where $w$ is a small window centered on $(i, j)$ in $A$ and $B$, respectively. $\sigma_{x}$ and $\sigma_{y}$ are the corresponding variances of two windows, and $\sigma_{x y}$ is the covariance between them. In Eq. 3, the first factor indicates the contrast similarity, and the second factor measures the degree of linear correlation at this location. In order to avoid being divided by zero, Eq. 3 is modified to

$$
\operatorname{DS}_{A B}(i, j)=\left.\frac{2\left|\sigma_{x y}\right|+C}{\sigma_{x}^{2}+\sigma_{y}^{2}+C}\right|_{w},
$$

where $C$ is a small constant. In Eq. (4), the size of the small window is set to $3 \times 3$, and this size is kept fixed in the following experiments. Larger window sizes will 
reduce the accuracy of comparisons. DS at location $(i, j)$ is figured out by averaging all the sub-DS that were computed at every NSCT directional band-pass subband pair in this location

$$
\operatorname{DS}_{A, B}(i, j)=\frac{\sum_{l=1}^{L} \sum_{k=1}^{L K} \mathrm{DS}_{H_{A}^{l, l k}, H_{B}^{l, l k}}(i, j)}{D},
$$

where $D$ is the total number of directional band-pass sub-bands. DS between $A$ and $B$ is the average of DS of all locations

$$
\operatorname{DS}_{A, B}=\frac{\sum_{i=1}^{M} \sum_{j=1}^{N} \operatorname{DS}_{A, B}(i, j)}{M \times N} .
$$

In Eq. (3), DS is measured without illumination comparison. This imitates an observed principle of HVS that humans would be more sensitive to the detail changes when objects are observed from close range.

\subsection{Color image evaluation}

For color images $A$ and $B$, the complementarity evaluation between them consists of the following steps:

(1) Compute VSMs $\operatorname{VSM}_{A}$ and $\mathrm{VSM}_{B}$ of $A$ and $B$.

(2) Transform $A$ and $B$ to HSV space.

(3) Compute the visual matching degree and DS of H, S and V components of each pixel $(i, j)$, respectively, obtaining $M_{A, B, H}(i, j), M_{A, B, S}(i, j), M_{A, B, I}(i, j)$, $\operatorname{DS}_{A, B, H}(i, j), \operatorname{DS}_{A, B, S}(i, j)$ and $\operatorname{DS}_{A, B, I}(i, j)$.

(4) The visual matching degree of each channel is defined as

$$
\begin{aligned}
M_{A, B, H}= & \frac{\sum_{j=1}^{N} \sum_{i=1}^{N} M_{A, B, H}(i, j)}{(M \times N)}, \\
M_{A, B, S}= & \frac{\sum_{j=1}^{N} \sum_{i=1}^{N} M_{A, B, H}(i, j)}{(M \times N)}, \\
M_{A, B, V}= & \frac{\sum_{j=1}^{N} \sum_{i=1}^{N} M_{A, B, V}(i, j)}{(M \times N)} .
\end{aligned}
$$

The whole $\operatorname{VSD}_{A, B}$ is computed as

$$
\mathrm{VSD}_{A, B}=w h \times M_{A, B, H}+w s \times M_{A, B, S}+w v \times M_{A, B, V} .
$$


(5) In the same way, DS of each channel and whole DS can be defined as

$$
\begin{aligned}
\mathrm{DS}_{A, B, H}= & \frac{\sum_{j=1}^{N} \sum_{i=1}^{N} \operatorname{DS}_{A, B, H}(i, j)}{(M \times N)}, \\
\operatorname{DS}_{A, B, S}= & \frac{\sum_{j=1}^{N} \sum_{i=1}^{N} \operatorname{Ds}_{A, B, S}(i, j)}{(M \times N)}, \\
\operatorname{DS}_{A, B, V}= & \frac{\sum_{j=1}^{N} \sum_{i=1}^{N} \operatorname{DS}_{A, B, V}(i, j)}{(M \times N)}, \\
\operatorname{DS}_{A, B}= & w h \times \operatorname{DS}_{A, B, H}+w s \times \operatorname{DS}_{A, B, S}+w v \times \operatorname{DS}_{A, B, V} .
\end{aligned}
$$

In Eqs. (8) and (9), weight coefficients $w_{h}, w_{s}$ and $w_{v}$ indicate the influence of different channels on the integrated result. They should satisfy

$$
w_{s}+w_{h}+w_{i}=1 .
$$

According to the research on the contrast sensitivity function of HVS, $\stackrel{3}{\text { human }}$ eyes are more sensitive to intensity than to color, and more sensitive to hue than to saturation, so we define $\left[w_{h}, w_{s}, w_{i}\right]=[0.15,0.1,0.75]$.

\section{Experiments and Discussion}

\subsection{Experimental setup}

To demonstrate the correctness and effectiveness of the proposed indices for the MST fusion quality evaluation and complementarity analysis, a large number of tests were carried out. 30 pairs of different types of fusion images were tested, including multi-focus images (10 pairs), infrared-visible images (six pairs), medical images (four pairs) and remote sensing images (10 pairs). In addition, several groups of color multi-exposure images are also used for color complementarity evaluations. The results of following tests are reported in the paper:

(1) Comparisons of the visual difference between different types of fused image pairs.

(2) Comparisons of general fusion rule-based MST fusion algorithms. These fusion algorithms employ DWT, SWT, DTCWT, CVT and NSCT as MST tools, respectively. For convenience of comparison, these algorithms employ the same general fusion rules. In this case, the average method was used for low-frequency coefficient fusion, and high-frequency coefficients were fused by choosing the absolute maximum. As verified in Ref. 17, the comparison results obtained for the general fusion rules were also valid for more powerful rules. 
(3) Comparisons of evaluation results of different NSCT filters and the number of decomposition levels.

(4) Complementarity evaluations. A group of color multi-exposure fusion images were used to analyze the complementarity between raw images and between raw images and their fusion results.

In the following tests, pyramid filter "pyrexc" and directional filter "7-9" were employed in NSCT first. The decomposition levels were set to $(4,8,8,16)$. This NSCT setting combination has been demonstrated to show the best performance in fusion algorithm comparisons in our work.

\subsection{Two-scale difference statistics of different types of raw images}

The differences between raw images vary widely for different fusion images. Four types of raw images were measured using the proposed two-scale metrics. Table 1 gives these statistical results.

In Table 1] we can see that, in coarse scale VSD comparisons, same-modality multi-focus image pairs have the smallest difference. By contrast, different-modality infrared-visible image pairs have the largest difference at the coarse scale. On the other hand, medical image pairs have the lowest DS clearly.

\subsection{Fusion quality evaluation using VSD and DS}

For fair comparison, the selection of optimal filters and decomposition levels for each MST fusion algorithm followed the research results of Ref. 17 (1) "db13" for DWT, four levels, (2) "bior2.2" for SWT, four levels, (3) "near symmetric "5-7" for the first level filter and "quarter sample shift orthogonal filter 10-10 with 6-6 nonzero taps" for the other level filters for DTCWT, four levels, (4) a two-dimensional FFTbased wrapping transform for CVT, four levels, (5) pyramid filter "pyrexc" and directional filter "7-9" for NSCT, $(4,8,8,16)$ levels. Four widely used evaluation indices, $Q, Q_{A B / F}, Q_{W}$ and $Q_{E}$ are contrasted with the proposed indices. These

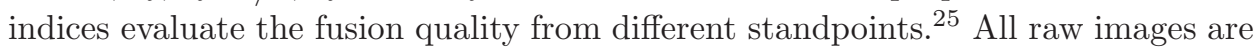
assumed to have been registered. Due to the limitation on page length, only the test results of multi-focus and remote sensing are reported below. Figure 3 shows a pair of multi-focus images (denoted as RI1 and RI2) and five fusion results.

Table 1. Two-scale difference statistical results of different types of raw images.

\begin{tabular}{lccc}
\hline Fusion type & Pairs & VSD range & DS range \\
\hline Multi-focus & 10 & $0.01-0.04$ & $0.65-0.90$ \\
Infrared-visible & 6 & $0.03-0.11$ & $0.35-0.65$ \\
Medical & 4 & $0.03-0.06$ & $0.25-0.35$ \\
Remote sensing & 10 & $0.01-0.05$ & $0.65-0.80$ \\
\hline
\end{tabular}




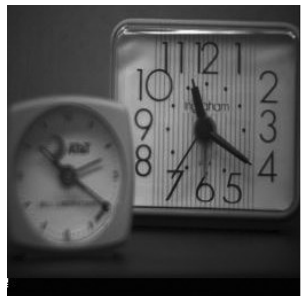

(a)

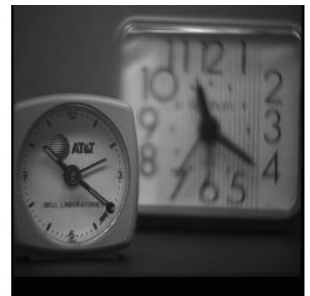

(b)

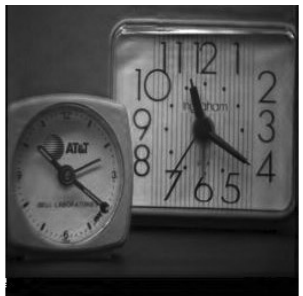

(c)

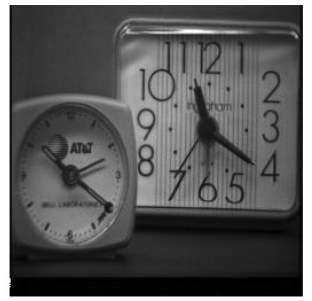

(d)

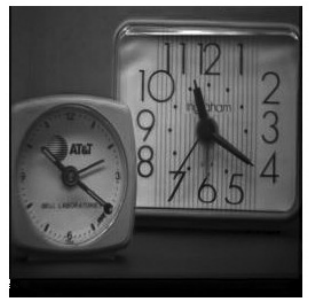

(e)

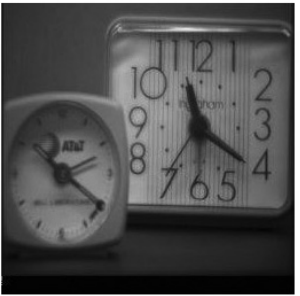

(f)

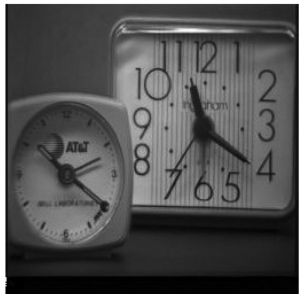

(g)

Fig. 3. Multi-focus fusion results. (a) RI1, (b) RI2, (c) DWT, (d) SWT, (e) DTCWT, (f) CVT and $(\mathrm{g}) \mathrm{NSCT}$.

Table 2. Comparison of multi-focus fusion results.

\begin{tabular}{|c|c|c|c|c|c|c|c|c|}
\hline & \multirow[b]{2}{*}{$Q$} & \multirow[b]{2}{*}{$Q_{A B / F}$} & \multirow[b]{2}{*}{$Q_{W}$} & \multirow[b]{2}{*}{$Q_{E}$} & \multicolumn{2}{|c|}{ VSD } & \multicolumn{2}{|c|}{ DS } \\
\hline & & & & & RI1/RI2 & Average & RI1/RI2 & Average \\
\hline DWT & 0.7763 & 0.6542 & 0.9274 & 0.7391 & $\begin{array}{l}0.0096 \\
0.0235\end{array}$ & $0.0166(\mathrm{~V})$ & $\begin{array}{l}0.8756 \\
0.8880\end{array}$ & $0.8816(\mathrm{~V})$ \\
\hline SWT & 0.8243 & 0.6990 & 0.9330 & 0.7427 & $\begin{array}{l}0.0126 \\
0.0062\end{array}$ & 0.0094 (III) & $\begin{array}{l}0.9072 \\
0.9072\end{array}$ & $0.9072(\mathrm{III})$ \\
\hline DTCWT & 0.8253 & 0.6946 & 0.9321 & 0.7313 & $\begin{array}{l}0.0123 \\
0.0060\end{array}$ & $0.0092(\mathrm{II})$ & $\begin{array}{l}0.9039 \\
0.9095\end{array}$ & $0.9067(\mathrm{IV})$ \\
\hline CVT & 0.8285 & 0.6376 & 0.8947 & 0.7347 & $\begin{array}{l}0.0149 \\
0.0066\end{array}$ & $0.0108(\mathrm{IV})$ & $\begin{array}{l}0.9293 \\
0.9134\end{array}$ & $0.9214(\mathrm{I})$ \\
\hline NSCT & 0.8371 & 0.7095 & 0.9373 & 0.7392 & $\begin{array}{l}0.0130 \\
0.0056\end{array}$ & $0.0083(\mathrm{I})$ & $\begin{array}{l}0.9090 \\
0.9162\end{array}$ & $0.9126(\mathrm{II})$ \\
\hline
\end{tabular}

Table 2 lists the testing results of multi-focus fusion. Here a smaller average VSD indicates a better fusion quality in the coarse scale. The fusion results that have higher average DS indicate stronger capabilities to extract detailed contents.

The results in Table 2 show that

(1) Two same-modality raw images have high similarity in coarse scale and low similarity in fine scale (their VSD is 0.0144 and DS is 0.3185 ). Five algorithms all get obvious improvements on DS. 
(2) NSCT algorithm has the smallest average VSD and the second-largest average DS compared to the other four. This demonstrates the capability of NSCT to achieve a more optimal decomposition.

(3) CVT gives the best average DS and relatively poor VSD metrics. This can be interpreted as the fact that CVT can capture more tiny curves and edges than other methods in this case. However, this advantage is diluted by the poor VSD metric when assessed using a single-scale index. This fact cannot be uncovered by the four single-scale indices listed in the table.

Figure 4 shows a pair of remote sensing multi-spectral images and the corresponding five fusion results.

Figures 4(a) and 4(b) show the raw remote sensing images with different spectrum ranges (denoted as RI1 and RI2). These different-modality images have

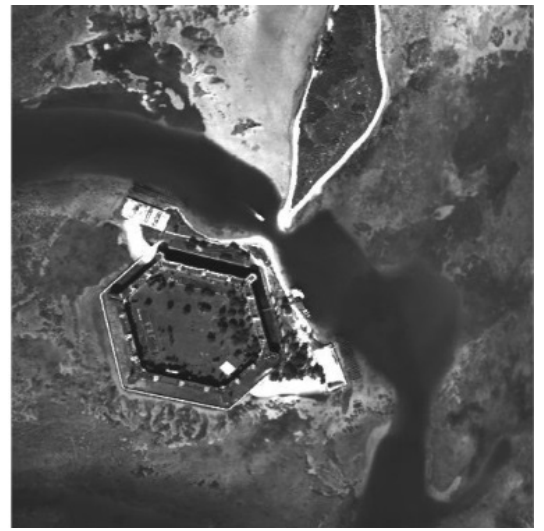

(a)

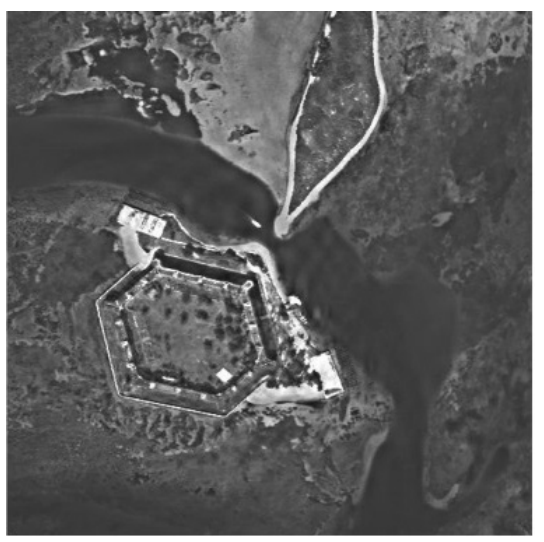

(c)

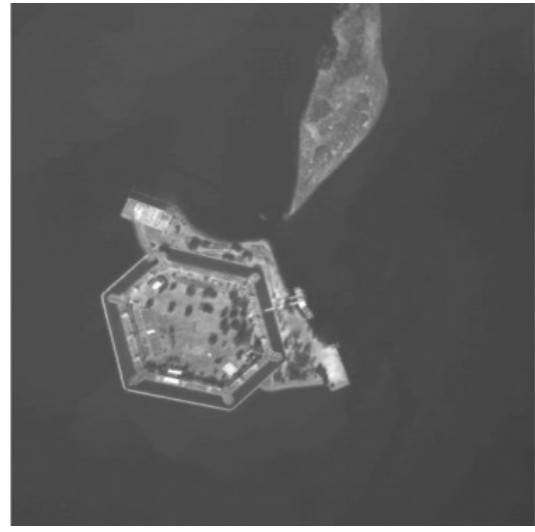

(b)

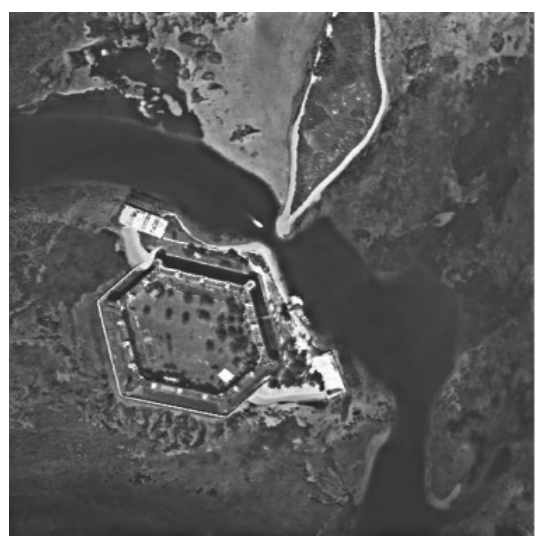

(d)

Fig. 4. Remote sensing fusion results. (a) RI1, (b) RI2, (c) DWT, (d) SWT, (e) DTCWT, (f) CVT and (g) NSCT. 


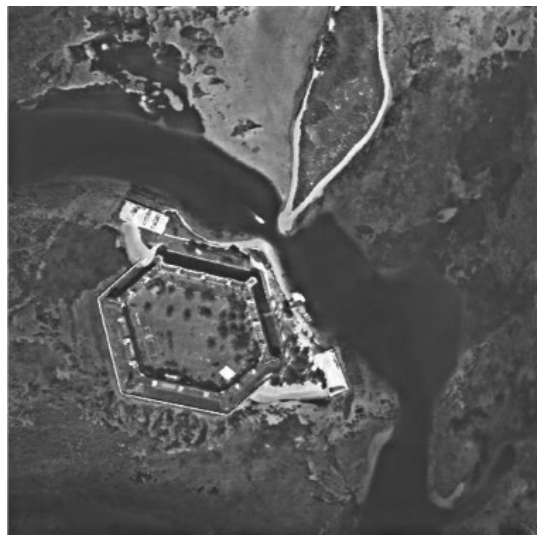

(e)

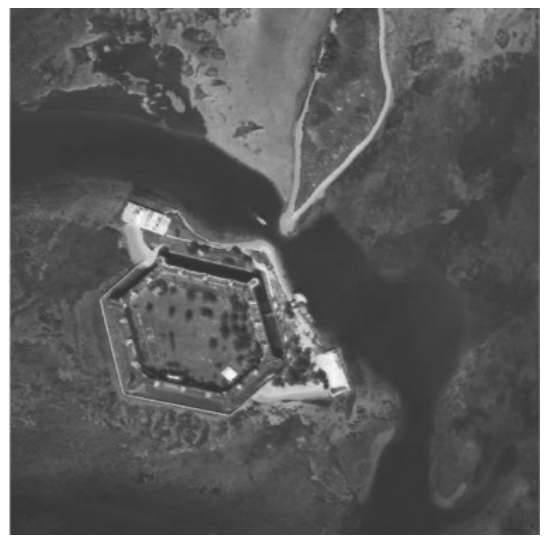

(f)

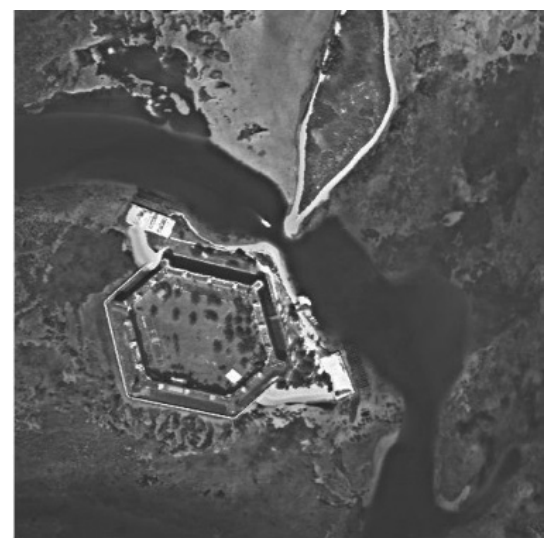

(g)

Fig. 4. (Continued)

large differences at two scales simultaneously, their VSD is 0.0554 and DS is 0.1663 . Figures 4(c) 4(g) show five fusion results respectively. Table 3 shows the results of this set of comparisons.

From this example, we can draw the following conclusions:

(1) Five algorithms have approximately equal VSD compared with two raw images, and RI2 contributes slightly more to fusion results. This complies with the average fusion rule used in low-frequency coefficient fusion.

(2) Because RI1 contains more details, the fusion results have higher DS with RI1 than with RI2. This complies with the maximum selection rule in highfrequency coefficient fusion.

(3) The NSCT algorithm outperforms the other algorithms in two scales for this type of fusion as well. 
Table 3. Comparison of remote sensing fusion results.

\begin{tabular}{|c|c|c|c|c|c|c|c|c|}
\hline & \multirow[b]{2}{*}{$Q$} & \multirow[b]{2}{*}{$Q_{A B / F}$} & \multirow[b]{2}{*}{$Q_{W}$} & \multirow[b]{2}{*}{$Q_{E}$} & \multicolumn{2}{|c|}{ VSD } & \multicolumn{2}{|c|}{$\mathrm{DS}$} \\
\hline & & & & & RI1/RI2 & Average & RI1/RI2 & Average \\
\hline DWT & 0.0844 & 0.7725 & 0.6210 & 0.8887 & $\begin{array}{l}0.0270 \\
0.0311\end{array}$ & $0.0291(\mathrm{IV})$ & $\begin{array}{l}0.8405 \\
0.2202\end{array}$ & $0.5304(\mathrm{~V})$ \\
\hline SWT & 0.0713 & 0.7981 & 0.6521 & 0.8966 & $\begin{array}{l}0.0237 \\
0.0325\end{array}$ & $0.0281(\mathrm{II})$ & $\begin{array}{l}0.8829 \\
0.2328\end{array}$ & $0.5579(\mathrm{II})$ \\
\hline DTCWT & 0.0693 & 0.8077 & 0.6618 & 0.8991 & $\begin{array}{l}0.0238 \\
0.0327\end{array}$ & $0.0282(\mathrm{III})$ & $\begin{array}{l}0.8666 \\
0.2305\end{array}$ & $0.5486(\mathrm{III})$ \\
\hline $\mathrm{CVT}$ & 0.1037 & 0.6582 & 0.5218 & 0.8332 & $\begin{array}{l}0.0296 \\
0.0281\end{array}$ & $0.0299(\mathrm{~V})$ & $\begin{array}{l}0.8046 \\
0.2855\end{array}$ & $0.5451(\mathrm{IV})$ \\
\hline NSCT & 0.0668 & 0.8212 & 0.6756 & 0.9041 & $\begin{array}{l}0.0218 \\
0.0339\end{array}$ & $0.0279(\mathrm{I})$ & $\begin{array}{l}0.9112 \\
0.2378\end{array}$ & $0.5745(\mathrm{I})$ \\
\hline
\end{tabular}

Table 4. A comprehensive ranking of the five fusion algorithms.

\begin{tabular}{|c|c|c|c|c|c|c|c|c|}
\hline & \multicolumn{2}{|c|}{ Multi-focus } & \multicolumn{2}{|c|}{ Medical } & \multicolumn{2}{|c|}{ Infrared-visible } & \multicolumn{2}{|c|}{ Remote-sensing } \\
\hline & VSD & DS & VSD & DS & VSD & DS & VSD & DS \\
\hline DWT & V & $\mathrm{V}$ & III & $\mathrm{V}$ & IV & $\mathrm{V}$ & IV & $\mathrm{V}$ \\
\hline SWT & IV & IV & IV & II & II & II & II & II \\
\hline DTCWT & III & III & II & IV & III & III & III & III \\
\hline CVT & II & I & $\mathrm{V}$ & III & $\mathrm{V}$ & IV & $\mathrm{V}$ & IV \\
\hline NSCT & I & II & I & I & I & I & I & I \\
\hline
\end{tabular}

(4) In contrast to the above multi-focus example, CVT obtains poor results for both VSD and DS metrics. This conclusion also agrees with four single-scale indices. Although CVT extracts more details from RI2 compared with the other four, at the same time it shows a poor extraction capability when it deals with RI1.

To evaluate the overall performance of five MST algorithms for different types of fusion, we counted the test results of each algorithm and present a comprehensive quality ranking in Table 4

Given above two-scale fusion performance comparison results, we can draw the following conclusions:

(1) A large difference exists between different fusion types of raw images. Multifocus fusion images have the smallest difference in detail scale, while the remote sensing fusion images show the biggest difference. Tables 1 and 2 also show that one fusion result will show a different DS when compared with RI1 and RI2, respectively.

(2) NSCT-based algorithms show the best performance in all fusion types. The above two-scale comparisons demonstrate that NSCT is the best MST tool for image fusion till now. 
(3) Three non-subsampling MSTs, including SWT, DTCWT and NSCT, also exhibit good performances for different-modality fusion. Shift-variance DWT and CVT perform comparatively poorly.

(4) CVT uses the superposition of bases that have variable shapes to represent the curves and edges, so it a exhibits a stronger capability to extract and reconstruct the image details. This fact has been clearly demonstrated in multi-focus fusion tests.

(5) Compared to four commonly used single-scale indices, our two-scale metrics can distinguish the "extraction capability of different scales" of the fusion algorithms effectively.

\subsection{Selections of filters and decomposition levels of NSCT}

Below, we assess the effects of different NSCT combinations of filters and decomposition levels on complementarity evaluations. At first, all 30 groups of raw images are fused with the optimal NSCT setting [pyramid filter "pyrexc", directional filter "7-9" and $(4,8,8,16)$ levels]. Then these fusion results are evaluated by using different NSCT setting combinations that consisted of four pyramid filters, 12 directional filters and four decomposition levels $[(4,8),(4,8,8),(4,8,8,8)$ and $(4,8$, $8,16)]$. By comparing these test results, we draw the following conclusions:

(1) Pyramid filters, which extract regional contents at coarse scales, have very little effect on VSD measurements, given the same directional filters and decomposition levels.

(2) Directional filters have a slight effect on detail extraction when other NSCT settings are fixed. The average ratio of the DS standard deviation is less than $0.4 \%$. The "7-9", "pkva", "McClellan" and "9-9" directional filters obtain slightly higher DS values given the same pyramid filter and decomposition level.

(3) The number of decomposition levels has opposite effects on VSD and DS. When decomposition levels increase, VSD will decrease and DS will increase. When scale decomposition level is set to 4 , more decomposition directions will bring very little effect on DS. When decomposition settings drop from $(4,8,8,16)$ to $(4,8)$, the average increasing ratio is about $3.6 \%$ for VSD and $-4.3 \%$ for DS. This inverse relationship between the number of decomposition levels and VSD is caused by the low-pass effects and noise sensitivity.

(4) The setting combination "pyrexc" + "7-9" + $(4,8,8,16)$ of NSCT presents the best assessing effects.

\subsection{Complementarity evaluations with VSD and DS}

In addition to fusion quality evaluation, this two-scale method can also be used for the difference comparisons of fused images. A group of multi-exposure gray-scale images shown in Fig. [5are used to compare visual complementarity, and then their color versions are used to demonstrate the color performance of our method. 


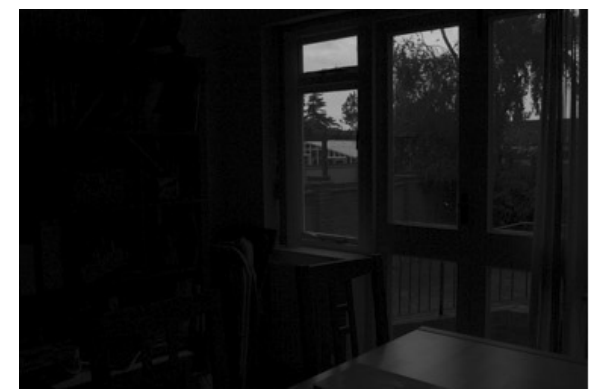

(a)

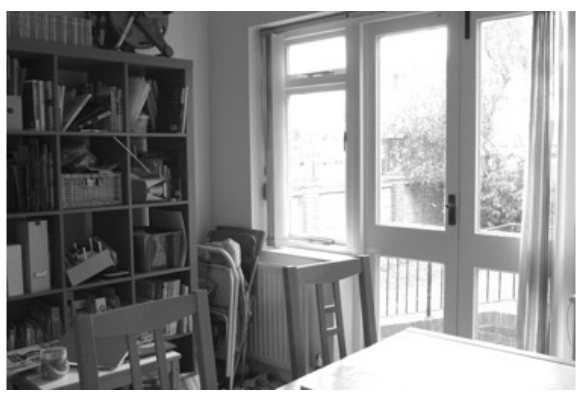

(c)

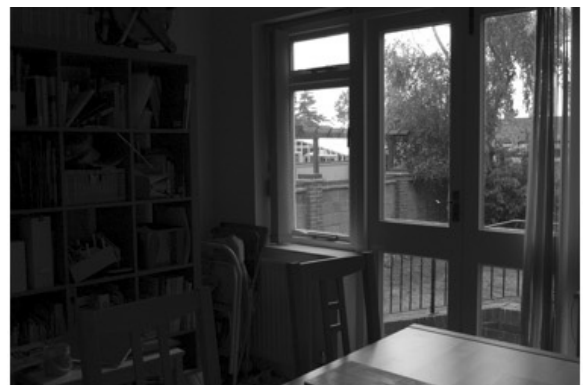

(b)

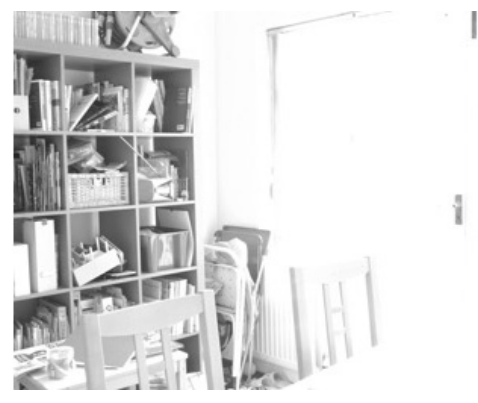

(d)

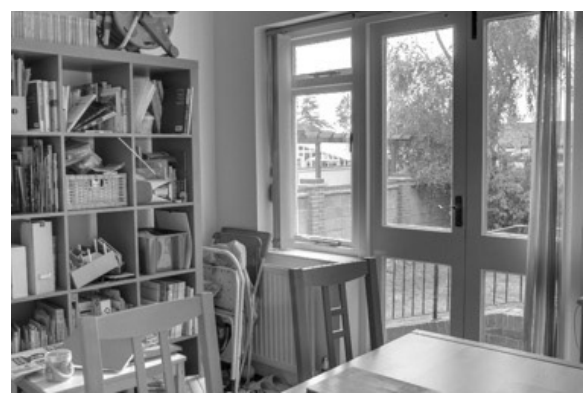

(e)

Fig. 5. Gray multi-exposure images and fusion results. 5 a) 5 d) four raw gray images, (e) the fusion result.

Figures 5 (a) 5 (d) show four raw gray-scale images, and Fig. 5(e) is the fusion result that is generated using the method proposed in Ref. 22. For the sake of brevity, these images are abbreviated as RI1, RI2, RI3, RI4 and FR, respectively. The average luminance values of these 8-bit gray-scale maps are 12.2, 49.7, 135.4, 223 and 127, respectively. In the following experiments, we take RI1 as a reference to compare the complementarity between raw images first, and then we take FR as a reference to compare the complementarity between their fusion result and raw images. Table 5 lists the test results between RI1 and the other three. 
Table 5. Complementarity comparisons of gray-scale multiexposure images $(1 / 2)$.

\begin{tabular}{lcccc}
\hline Compared groups & Average luminance & VSD & DS \\
\hline RI1 & vs RI2 & $12.2 / 49.7$ & 0.0430 & 0.3227 \\
& vs RI3 & $12.2 / 135.4$ & 0.1568 & 0.2141 \\
& vs RI4 & $12.2 / 223$ & 0.2723 & 0.1134 \\
\hline
\end{tabular}

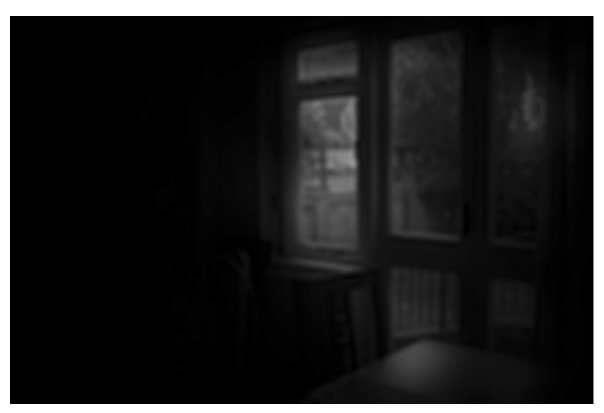

(a)

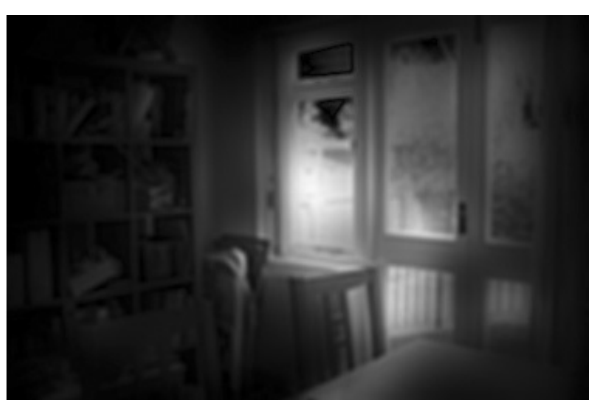

(b)

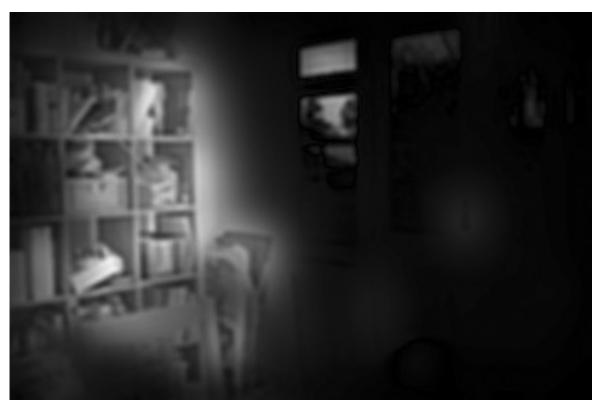

(c)

Fig. 6. Maps of VSD of gray-scale multi-exposure images. (a) VSD map of RI1 vs. RI2, (b) VSD map of RI1 vs. RI3, (c) VSD map of RI1 vs. RI4.

We can note that VSD increases rapidly as the average luminance difference increases. This indicates the increases of dissimilar regions at coarse scale. In contrast, DS decreases because of the decreasing overlapped regions. The VSD maps of each compared group are shown in Fig. 6

From Fig. 6, we can see that with the increases of luminance, the well-exposed region transfers from the right to the left. Hence, compared to RI1, raw images RI2, RI3 and RI4 have incremental visual complementarities, as indicated in Table 4.

Beyond these comparisons, the distributions of visual complementarity between raw images can also be quantitatively analyzed from Table 5 . It can be noted that as the average illumination of these raw images increases equidistantly, VSD and DS also vary equidistantly. This means that the change amplitudes of VSD and 


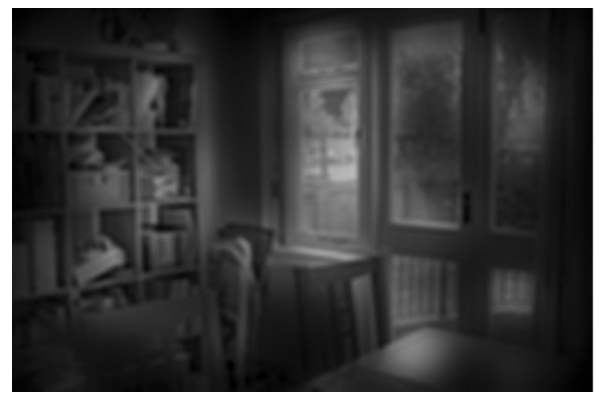

(a)

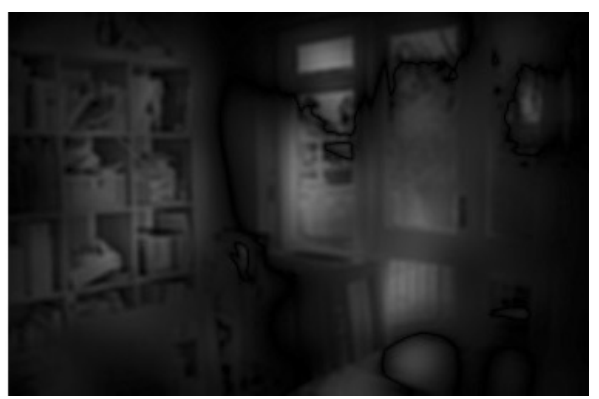

(c)

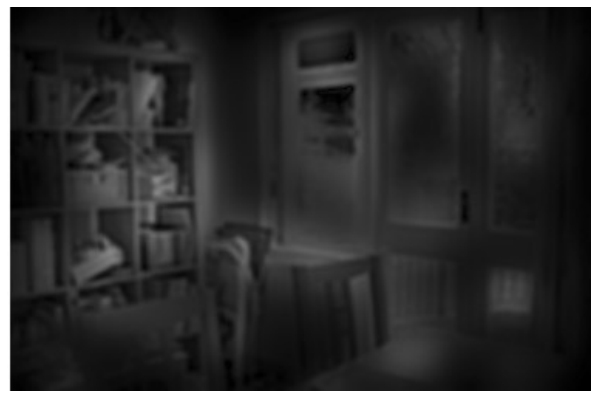

(b)

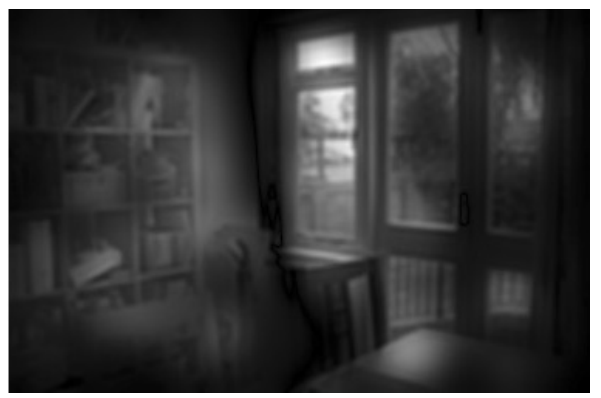

(d)

Fig. 7. VSD comparison between the fusion result and raw images. (a) VSD Map of FR vs. RI1, (b) VSD Map of FR vs. RI2, (c) VSD Map of FR vs. RI3 and (d) VSD Map of FR vs. RI4.

DS are approximately linearly proportional to the illumination change amplitudes. Hence we can conclude that in multi-exposure fusion, the raw images should be equidistantly distributed in the camera's illumination space and cover the dynamic range as much as possible.

Next we take the FR shown in Fig. [5) (e) as a reference to the comparisons of visual complementarity between raw images and fusion their results. Figure[7]shows these resulting VSD maps.

It is clear that Figs. 7(a) and 7(d) have a larger average intensity than Figs. 7(b) and 7 c). This means that FR has extracted more information from RI2 and RI3 than from RI1 and RI4. The reason is that the employed fusion algorithm assigns higher priority to the regions with middle intensity than the under-exposed and over-exposed regions. Table 6 lists the visual complementarity between FR and raw images.

In Table 6. RF has a smaller VSD and a larger DS with RI3 and RI2 than with RI1 and FR4. This indicates that FR collects more details from RI3 and RI2. This conclusion accords with that of Fig. 7 .

Figure 8 shows the raw color images and their fusion results (abbreviated as RI1, RI2, RI3, RI4 and FR, respectively). 
A bio-inspired two-scale complementarity evaluation method for image fusion

Table 6. Complementarity comparisons of gray multi-exposure images (2/2).

\begin{tabular}{|c|c|c|c|c|}
\hline \multicolumn{2}{|c|}{ Compared groups } & \multirow{2}{*}{$\begin{array}{c}\text { Average luminance } \\
127 / 12.2\end{array}$} & \multirow{2}{*}{$\frac{\text { VSD }}{0.1629}$} & \multirow{2}{*}{$\frac{\mathrm{DS}}{0.2415}$} \\
\hline FR & vs RI1 & & & \\
\hline & vs RI2 & $127 / 49.7$ & 0.1200 & 0.5446 \\
\hline & vs RI3 & $127 / 135.4$ & 0.0925 & 0.6777 \\
\hline & vs RI4 & $127 / 223$ & 0.1698 & 0.4436 \\
\hline
\end{tabular}

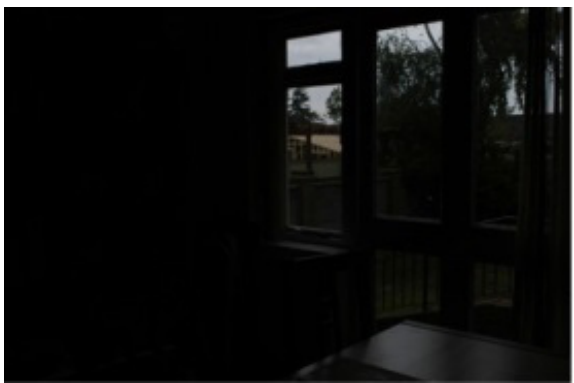

(a)

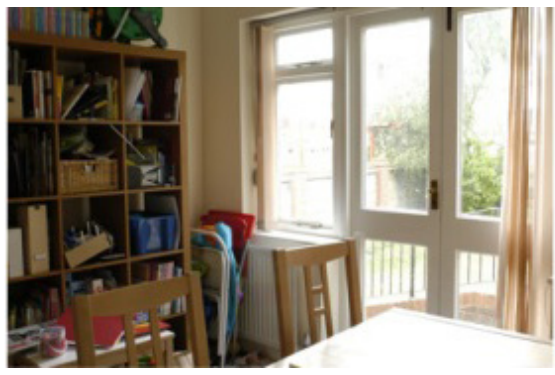

(c)

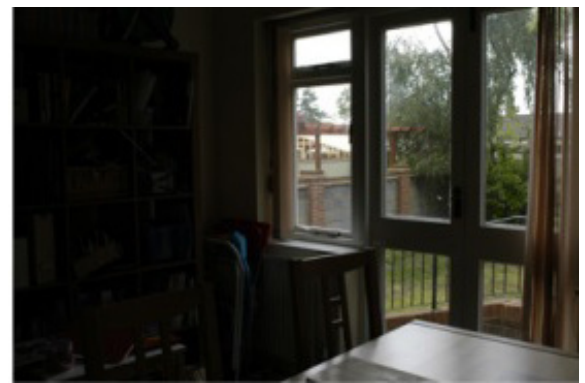

(b)

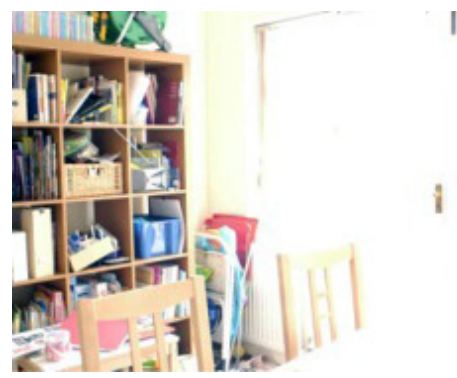

(d)

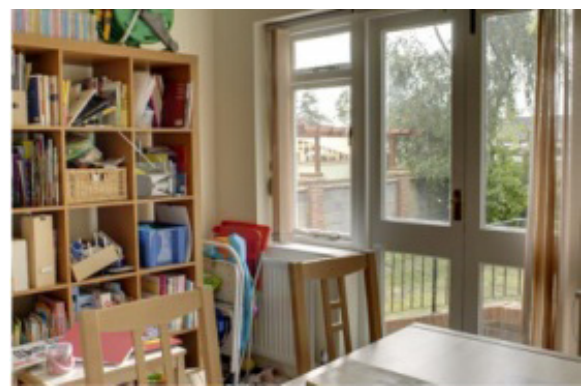

(e)

Fig. 8. Color multi-exposure images and their fusion results. 8 (a) 8 (d) four raw images, 8 e) the fusion result. 
Table 7. Complementarity comparisons of color multi-exposure images $(1 / 2)$.

\begin{tabular}{|c|c|c|c|c|c|c|c|c|c|}
\hline \multicolumn{2}{|c|}{$\begin{array}{l}\text { Compared } \\
\text { groups }\end{array}$} & \multirow{2}{*}{$\frac{M_{A, B, H}}{0.0416}$} & \multirow{2}{*}{$\frac{M_{A, B, S}}{0.0935}$} & \multirow{2}{*}{$\frac{M_{A, B, V}}{0.0346}$} & \multirow{2}{*}{$\begin{array}{c}\text { Whole } \\
\text { VSD }\end{array}$} & \multirow{2}{*}{$\frac{\mathrm{DS}_{A, B, H}}{0.1762}$} & \multirow{2}{*}{$\frac{\mathrm{DS}_{A, B, S}}{0.1707}$} & \multirow{2}{*}{$\frac{\mathrm{DS}_{A, B, V}}{0.1679}$} & \multirow{2}{*}{$\begin{array}{c}\text { Whole } \\
\text { DS }\end{array}$} \\
\hline RI1 & vs RI2 & & & & & & & & \\
\hline & vs RI3 & 0.0585 & 0.1267 & 0.1676 & 0.1635 & 0.1549 & 0.1229 & 0.1438 & 0.1434 \\
\hline & vs RI4 & 0.0714 & 0.0908 & 0.1606 & 0.1536 & 0.1331 & 0.0723 & 0.0646 & 0.0756 \\
\hline
\end{tabular}

Note: Symbol definitions please refer to Sec. 3.3.

Table 8. Complementarity comparisons of color multi-exposure images $(2 / 2)$.

\begin{tabular}{|c|c|c|c|c|c|c|c|c|c|}
\hline \multicolumn{2}{|c|}{$\begin{array}{l}\text { Compared } \\
\text { groups }\end{array}$} & \multirow{2}{*}{$\begin{array}{c}M_{A, B, H} \\
0.0492\end{array}$} & \multirow{2}{*}{$\begin{array}{r}M_{A, B, S} \\
0.0914\end{array}$} & \multirow{2}{*}{$\frac{M_{A, B, V}}{0.1713}$} & \multirow{2}{*}{$\begin{array}{c}\text { Whole } \\
\text { VSD }\end{array}$} & \multirow{2}{*}{$\frac{\mathrm{DS}_{A, B, H}}{0.1197}$} & \multirow{2}{*}{$\frac{\mathrm{DS}_{A, B, S}}{0.1077}$} & \multirow{2}{*}{$\frac{\mathrm{DS}_{A, B, V}}{0.1637}$} & \multirow{2}{*}{$\begin{array}{c}\text { Whole } \\
\text { DS }\end{array}$} \\
\hline 17 & vs RI1 & & & & & & & & \\
\hline & vs RI2 & 0.0286 & 0.0703 & 0.0630 & 0.0637 & 0.2406 & 0.1814 & 0.1840 & 0.1923 \\
\hline & vs RI3 & 0.0221 & 0.0705 & 0.0494 & 0.0515 & 0.2775 & 0.2336 & 0.2134 & 0.2251 \\
\hline & vs RI4 & 0.0397 & 0.0674 & 0.1227 & 0.1172 & 0.2318 & 0.1662 & 0.1627 & 0.1734 \\
\hline
\end{tabular}

In this experiment, the image RI1 is first used as a reference for the comparisons of the complementarity between raw images. Then FR is used as a reference for comparisons of the fusion result and raw images. Table 7 lists the test results between raw images.

The change patterns of color complementarity between raw images can be summarized as follows:

(1) As the average illumination increases, hue and saturation increase first and then decrease when some over-exposed regions appear. In whole VSD comparisons, RI1 has the largest difference from RI3, rather than with RI4. This is caused by the large difference in saturation and hue between RI1 and RI3.

(2) DS of $H, S$ and $V$ channels decrease as the illumination average increases, and RI1 has the least DS with RI4. These fine scale comparison results accord with the gray image comparisons listed in Table 4 .

From Table [1, we can note that the distributions of color complementarity are not as intuitive as gray-scale images. This is due to the nonlinear features of HSV space. Table 8 lists the comparisons between FR and four raw images.

From Table 8 it can be noted that RI3 has the highest similarity with the fusion result. The reason for this is that the employed algorithm assigns larger weight values to the pixels with moderate brightness. So RI3, whose average intensity is close to 128 , contributes more information to the fusion result.

\section{Conclusion}

In this paper, a bio-inspired two-scale complementarity evaluation method for image fusion was presented. It opens up a new perspective for the assessment of multi-scale fusion algorithms. This novel two-scale method imitates the characteristics of HVS, 
and evaluates visual complementarity at two different spatial scales by employing VAM and NSCT. Five general rules-based MST fusion algorithms, DWT, SWT, DTCWT, CVT and NSCT, were tested and compared with four types of fusion images. Different NSCT setting combinations are also investigated in detail. In addition, an example of color complementarity evaluation is also presented in detail. Our main research conclusions are as follows:

(1) Of the different types of image fusion, the differences between raw images vary widely. We give numerical proofs that raw images with the same-modality have higher similarity at two scales simultaneously.

(2) Of the five investigated MST fusion algorithms, NSCT has the best global quality in all types of fusion images. Three shift-invariance MSTs, including NSCT, DTCWT and SWT, have evident advantages over CVT and DWT.

(3) CVT shows the strongest detail extraction capability in multi-focus image fusion. However, this advantage is encumbered by its poor coarse scale performance.

(4) NSCT pyramid filters and directional filters show limited influences on evaluation results. More decomposition levels would improve the detail comparison capability slightly, but at the same time restrain the coarse scale comparison capability.

(5) Two scale evaluation metrics uncover the contributions of raw image to the fusion result at different scales clearly. In multi-exposure image fusion, to obtain the best results, raw images should be equidistantly distributed in the illumination space and cover the dynamic range as widely as possible.

\section{Acknowledgments}

The authors would like to thank the anonymous reviewers for their valuable comments. We are grateful to Professor L. Itti and Minh N. Do for their work on the VAM and NSCT toolboxes, respectively. We would also like to thank Professors S. T. Li and B. Yang for their previous work on fusion algorithm evaluation.

This work was supported by the National Natural Science Foundation of China under Project Number 61274021.

\section{References}

1. D. R. Amol, V. J. Dattatray and S. H. Raghunath, Fast discrete curvelet transform based anisotropic iris coding and recognition using k-out-of-n: A fused post-classifier, Mach. Vis. Appl. 23(6) (2012) 1115-1127.

2. S. Arivazhagan, L. Ganesan and K. Subash, A modified statistical approach for image fusion using wavelet transform, Signal Image Video P. 3(2) (2009) 137-144.

3. P. G. J. Barten, Contrast Sensitivity of the Human Eye and Its Effects on Image Quality (SPIE Press Book, 1999).

4. A. Borji and L. Itti, State-of-the-art in visual attention modeling, IEEE Trans. Pattern Anal. Mach. Intell. 35(1) (2013) 185-207. 
5. M. Carrasco, Visual attention: The past 25 years, Vision Res. 51(13) (2011) 14841525.

6. M. N. Do, NSCT toolbox, http://minhdo.ece.illinois.edu/software/.

7. J. Dong et al., Advances in multi-sensor data fusion: Algorithms and applications, Sensors 9(10) (2009) 7771-7784.

8. J. Harel, Graph-based visual saliency, http://www.klab.caltech.edu/ harel/share/ gbvs.php.

9. Z. Y. He, X. G. You and Y. Yuan, Texture image retrieval based on non-tensor product wavelet filter banks, Signal Process. 89(8) (2009) 1501-1510.

10. X. D. Hou and L. Q. Zhang, Saliency detection: A spectral residual approach, in Proc. 2007 IEEE Conf. on Computer Vision and Pattern Recognition (IEEE, Minneapolis, MN, USA, 17-22 June, 2007), pp. 1-8.

11. Y. X. Hu and R. Zhang, Vision model based image fusion in nonsubsampled contourlet transform domain, in Proc. IEEE 11th Conf. on Industrial Electronics and Applications (IEEE, Hefei, China, 5-7 June, 2016), pp. 7603780.

12. L. Itti, Models of bottom-up and top-down visual attention, Ph.D. thesis, California Institute of Technology, Pasadena, California, USA (2000). This thesis can be downloaded from: http://thesis.library.caltech.edu/4722/.

13. A. P. James and B. V. Dasarathy, Medical image fusion: A survey of the state of the art, Inf. Fusion. 19(3) (2014) 4-19.

14. W. W. Kong, Y. J. Lei and Y. Lei, Image fusion technique based on non-subsampled contourlet transform and adaptive unit-fast-linking pulse-coupled neural network, IET Image Process. 5(2) (2011) 113-121.

15. S. T. Li, J. T. Kwok and Y. N. Wang, Discrete wavelet frame transform method to merge Landsat TM and SPOT panchromatic images, Inf. Fusion 3(1) (2002) 17-23.

16. T. J. Li and Y. Y. Wang, Biological image fusion using a NSCT based variable-weight method, Inf. Fusion 12(2) (2011) 85-92.

17. S. T. Li, B. Yang and J. Hu, Performance comparison of different multi-resolution transforms for image fusion, Inf. Fusion 12(2) (2011) 74-84.

18. Z. Liu and E. Blasch, Statistical analysis of the performance assessment results for pixel-level image fusion, in 17th Int. Conf. on Inf. Fusion (IEEE, Salamanca, Spain, 7-10 July, 2014), pp. 1-8.

19. Z. Liu, E. Blasch, Z. Xue, R. Langaniere and W. Wu, Objective assessment of multiresolution image fusion algorithms for context enhancement in night vision: A comparative survey, IEEE Trans. Pattern Anal. Mach. Intell. 34(1) (2012) 94-109.

20. J. G. Liu, Smoothing filter based intensity modulation: A spectral preserve image fusion technique for improving spatial details, Int. J. Remote Sens. 21(18) (2000) 3461-3472.

21. K. Liu, L. Guo, W. Chang and H. Li, Algorithm of image fusion based on finite ridgelet transform, in Proc. 2005 IEEE Int. Symp. on Geoscience and Remote Sensing (SPIE, Wuhan, China, November 15, 2007), pp. 1150-1153.

22. T. Mertens, J. Kautz and F. V. Reeth, Exposure fusion, in Proc. 2007 Int. Conf. on Pacific Graphics (IEEE, Maui, HI, USA, 29 October-2 November, 2007), pp. 382-390.

23. S. C. Nercessian, K. A. Panetta and S. Agaian, Multiscale image fusion using an adaptive similarity-based sensor weighting scheme and human visual system-inspired contrast measure, J. Electron. Imaging 21(2) (2012) 021112.

24. W. Ou et al., Robust face recognition via occlusion dictionary learning, Pattern Recognit. 47(4) (2014) 1559-1572.

25. G. Piella and H. Heijmans, A new quality metric for image fusion, in Proc. IEEE Int. Conf. on Image Processing (IEEE, Barcelona, Spain, 2003), pp. 173-176. 
26. R. Singh and A. Khare, Fusion of multimodal medical images using Daubechies complex wavelet transform - A multi-resolution approach, Inf. Fusion 19(3) (2014) 49-60.

27. A. Toet et al., Towards cognitive image fusion, Inf. Fusion. 11(2) (2010) 95-113.

28. Z. Wang and A. C. Bovik, A universal image quality index, IEEE Signal Proccess. Lett. 9(3) (2002) 81-84.

29. J. Wang et al., Image fusion with non-subsampled contourlet transform and sparse representation, J. Electron. Imaging. 22(4) (2013) 04301901.

30. S. Y. Yang et al., Image fusion based on a new contourlet packet, Inf. Fusion 11(2) (2010) 78-84.

31. X. G. You et al., A blind watermarking scheme using new non-tensor product wavelet filter banks, IEEE Trans. Image Process. 19(12) (2012) 3271-3284.

32. L. N. Yu, S. P. Liu and Z. F. Wang, Multi-focus image fusion with dense SIFT, Inf. Fusion 23 (2015) 139-155.

33. S. Yu et al., A new algorithm for shoreline extraction from satellite imagery with non- separable wavelet and level set method, Int. J. Mach. Learn. Comput. 3(1) (2013) 158-163. 